\title{
Practical Aspects in Topical PUVAsol in Dermatology: An Experience in a Teaching Hospital
}

\section{KC S, Karn D}

Department of Dermatology

Dhulikhel Hospital, Kathmandu University Hospital

Dhulikhel, Kavre, Nepal

Corresponding Author

Dharmendra Karn

Department of Dermatology

Dhulikhel Hospital, Kathmandu University Hospital

Dhulikhel, Kavre, Nepal

E-mail: dddkarn@gmail.com

Citation

KC S, Karn D. Practical Aspects in Topical PUVAsol in Dermatology: An Experience in a Teaching Hospital. Kathmandu Univ Med J 2014;48(4):306-7.

\section{INTRODUCTION}

Psoralen ultraviolet A (PUVA) is a proven modality in dermatological therapeutics. It is widely practiced in the treatment of psoriasis, vitiligo, atopic eczema, certain skin cancers, photosensitivity dermatoses, alopecia areata, graft-versus-host disease and morphea. ${ }^{1-3}$ In presence of UVA, psoralen intercalates between the DNA base pairs forming functional adducts, free radicals and reactive oxygen species thus causing cross linking of DNA strands, protein conjugation and cytotoxic effects.

Depending upon source of UVA, the therapy can be given as PUVA (artificial phototherapy unit as the source of UVA) or PUVAsol (solar irradiation as the source of UVA). Topical psoralen containing preparations are safer and more convenient as compared to oral psoralen. ${ }^{4-5}$ Bath PUVA and other topical forms of PUVA therapy offers number of advantages over systemic PUVA. Better compliance is achieved because of negligible nausea, ocular or central nervous system effects and lower cumulative UVA dose. Topical PUVA is preferable to systemic PUVA especially in settings of hepatic or gastro-intestinal dysfunction, cataract, poor eye protection and anticipated drug interaction. ${ }^{1}$ Sunlight is an abundant source of UVA almost throughout the year in Nepal. Here PUVAsol is an appropriate and inexpensive regimen especially for patients who cannot visit hospital frequently and those with economic constraints. Hence, we intend to discuss the various modalities of topical PUVAsol therapy and our experience as feasible in the context of Nepal. In view of short communication we do not present any forms of data in this subset.

\section{Practical aspects of topical PUVAsol}

Soak PUVAsol, paint PUVAsol and bath PUVAsol are the various modalities employed for topical PUVAsol therapy. These therapies are generally practiced twice a week. Soak and paint PUVAsol are the commonly applied methods. These therapies are prescribed on twice a week basis. Such forms of therapy are generally helpful for limited plaque psoriasis, palmoplantar psoriasis, hand-foot eczema and to induce pigmentation following vitiligo surgery. In soak PUVAsol, hands and/or feet are soaked in a tub containing $0.03 \%$ methoxsalen for $20-30$ minutes. ${ }^{5}$ The desired concentration is achieved by dissolving one tablet of $10 \mathrm{mg}$ methoxsalen or $1 \mathrm{~mL}$ of $1 \%$ solution in 3 litres of water. The temperature of water can vary according to patient preference. The same concentration of solution can be used to treat scalp diseases such as scalp psoriasis and alopecia areata. Soaking and re-soaking a towel periodically with the medicated solution and placing it in the scalp in form of a turban for 10-15 minutes has been practiced. ${ }^{6}$ A tight cotton cap can be used alternatively. The concerned site is subjected to solar irradiation within 15 to 20 minutes which is started at 1-2 minutes initially. The duration of sun exposure is then gradually increased (one minute per week) until maximum 10-15 minutes or until perceptible erythema. As most of our patients may remain outdoor throughout the day, exposure in the morning may result in excessive cumulative UVA and ocular toxicity. For this reason we prefer to prescribe PUVAsol between 2 p.m. to $3: 30$ p.m.

Bath PUVAsol is not practically feasible because of the unavailability of bathtub. Hence for patients requiring bath PUVAsol we prescribe bathing suit PUVAsol technique as described by Pai $S$ et al. ${ }^{7}$ Tight clothing resembling a bath suit is ideal for this purpose. The cloth is soaked in the solution and applied over the body similarly as turban PUVAsol at a concentration of $0.000075 \%$ prior to irradiation (example: one tablet methoxsalen in 13-14 litres water). We are currently continuing PUVAsol therapy and the outcome has been rewarding. However the data collection is ongoing for its future validation.

\section{DISCUSSION}

In PUVA, TMP (Trimethylpsoralen, trioxsalen) is preferable to 8-MOP due to its relative weaker penetrability and phototoxic effects. ${ }^{8}$ PUVAsol is generally offered twice to thrice weekly, with a minimum gap of 1 day between treatments. No general guidelines have been developed regarding the use of PUVAsol. Gahalaut $P$ et al proposed 
to begin PUVAsol at 5 minutes per day of solar irradiation every alternate day. ${ }^{9}$ Subsequent exposure until maximum of 30 minutes was reached. Similarly, Sornakumar $L$ et al suggested turban PUVAsol at 5 minutes per exposure up to maximum of 15 minutes. ${ }^{6}$ However we noticed few photosensitive events at a higher initial dose. Balasaraswathy $P$ et al had recommended the ideal time for PUVAsol to be between 9.30 a.m. to 11 a.m. or 2 p.m. to 3.30 p.m. when the incidental UVB and infrared rays responsible for heat are less. ${ }^{10}$

In our center, we use soak PUVAsol and bath suit PUVAsol with tablets of methoxsalen. No undesired adverse effects have been recorded with this therapy. Paint PUVAsol has been found to have higher frequency of erythema and blistering which might be because of the difficulty for patient in maintaining dilution. A comparative study between PUVA versus PUVAsol among patients with vitiligo indicated that PUVA is more efficacious and also provides a greater psychological benefit than PUVAsol. ${ }^{9}$ Nevertheless the phototoxic side effects were found to be significantly greater among patients treated with PUVA therapy. However; lack of definite controlled parameters, noncompliance, privacy issues are some of its disadvantages. Also the incident UVA varies according to season, time of the day, latitude and atmospheric conditions. Exposure to solar radiation at noon time may further lead to the unwanted effects of ultraviolet B (UVB). UVB from the sunlight can increase the epidermal thickness and may interfere with the effectiveness of the therapy. PUVAsol can even be carried out following oral proralen. However distressing nausea, headache and ocular toxicity are the possible disadvantages.

Time consuming labour, development of erythema, blisters and irregular pigmentation at treatment site are some probable side effects of topical PUVAsol. Still then avoidance of the systemic side effects remains to be the greatest asset of topical PUVAsol. Pregnancy, lactation, pediatric population and patients with photosensitivity diseases are common contraindications of the therapy. Various combination therapies have also been described along with PUVAsol which can help the chronicity of different diseases to remain under remission.

\section{CONCLUSION}

Topical PUVAsol offers a number of advantages of being cheap, using locally available resources and being free from the systemic adverse effects in comparison to systemic therapies in resource poor settings. However, further studies are needed in the future to validate the efficacy of PUVAsol therapy.

\section{REFERENCES}

1. Halpern SM, Anstey AV, Dawe RS, Diffey BL, Farr PM, Ferguson J et al. Guidelines for topical PUVA: a report of a workshop of the British photodermatology group. Br J Dermatol 2000;142(1):22-31.

2. Shenoi SD, Prabhu S. Photochemotherapy (PUVA) in psoriasis and vitiligo. Indian J Dermatol Venereol Leprol 2014;80(6):497-504.

3. Srinivas CR, Pai S. Psoralens. Indian I Dermatol Venereol Leprol 1997;63(5):276-87.

4. Rai R, Srinivas CR. Phototherapy: An Indian Perceptive. Indian J Dermatol 2007;52(4):169-75.

5. Tsui $\mathrm{CL}$, Levitt J. Practical pearls in phototherapy. Int I Dermatol 2013;52(11):1395-7.

6. Sornakumar L, Sekar CS, Srinivas CR. Turban PUVASOL: An Effective Treatment in Alopecia Totalis. Int J Trichology 2010;2(2):106-7.

7. Pai S, Srinivas CR. Bathing suit delivery of 8-methoxy psoralen for psoriasis: A double blind, placebo-controlled study. Int J Dermatol 1994;33(8):576-8.

8. Said A, Makki S, Muret $P$, Humbert $P$, Millet J. Psoralens percutaneous permeation across the human whole skin and the epidermis in respect to their polarity (in vitro study). J Dermatol Sci 1997;14(2):136-44.

9. Gahalaut P, Mishra N, Soodan PS, Rastogi MK. Effect of Oral PUVAsol on the Quality of Life in Indian Patients Having Chronic Plaque Psoriasis. Dermatol Res Pract 2014;2014:291586.

10. Balasaraswathy $P$, Kumar $U$, Srinivas $C R$, Nair S. UVA and UVB in sunlight, Optimal Utilization of UV rays in Sunlight for phototherapy. Indian J Dermatol Venereol Leprol 2002;68(4):198-201. 